\title{
Promoción de la salud en Cuba ${ }^{1}$
}

Health promotion in Cuba Promoção da saúde em Cuba

Elena Díaz ${ }^{2}$

\section{RESUMEN}

El texto refiere los antecedentes de la situación de la salud en Cuba, presentando la caracterización del sistema actual de salud, con los niveles que lo integran, en particular la atención primaria, y los ajustes que se han introducido; también destaca la presencia de cuatro programas priorizados, de los cuales se presentan algunos de sus rasgos mas significativos. Estos son la atención materno infantil, el control de enfermedades trasmisibles, incluyendo el programa para prevenir el VIH; el control de enfermedades no trasmisibles, y el programa de atención al adulto mayor. Se hace referencia al marco conceptual sobre Atención Primaria y Promoción de salud, enfatizando la plataforma social que permite instrumentar en Cuba las políticas universales simultáneamente a las focalizadas basadas en cuatro fortalezas de la sociedad: la tendencia a garantizar la equidad y la justicia social, las bases creadas del acceso a la educación, la interrelación del desarrollo del conocimiento científico y su aplicación a la salud de la población, y la promoción permanente de valores éticos solidarios. Finalmente, se hace referencia a los modelos de formación que se instrumentan en la actualidad.

PALABRAS CLAVE: Atención Primaria; Promoción de Salud.

\section{ABSTRACT}

This paper looks at the historical background of the health situation in Cuba by characterizing the current health system; the levels that make part of it, in particular primary health care; the adjustments introduced, and also highlights the existence of four prioritized programs which present some of its most significant features. Those are: mother and child care; transmissible disease control including the HIV prevention program; non-transmissible disease control and care for the elderly program. Reference is made to the conceptual landmark in Primary Care and Health Promotion. Here we

\footnotetext{
${ }^{1}$ Este texto fue preparado en base a la ponencia presentada en el I Seminario Internacional de Estudios Comparados, convocado por el Núcleo de Estudios de Salud Pública, Universidad de Brasilia, Brasil, diciembre 2008.

${ }^{2}$ Licenciada en Filosofía y Letras, Doctora en Ciencias Económicas. Profesora Titular de La Universidad de La Habana. Profesora Titular. Programa FLACSO Cuba, adscrito a la Universidad de La Habana. (U.H.)

Tempus - Actas de Saúde Coletiva, vol. 3, no2. p. 99-111, Abr. / jun.
} 
highlight the social platform that allows Cuba to implement universal policies simultaneously to those based on the four tenants of society: tendency to secure equity and social justice; the basis of access to education; the interrelationship between scientific knowledge development and its application to population health and the permanent promotion of ethical solidarity values. Lastly, reference is made to the models of formation currently implemented.

KEYWORDS: Primary Care, Health Promotion.

\section{RESUMO}

O texto se refere aos antecedentes da situação da saúde em Cuba, apresentando a caracterização do sistema atual de saúde, com os níveis que o integram, em particular a atenção primária, e os ajustes que se tem introduzido; também destaca a presença de quatro programas priorizados, dos quais se apresentam alguns de seus rasgos mais significativos. Estes são: a atenção materna infantil, o controle de doenças transmissíveis, incluindo o programa para prevenção do VIH; o controle de doenças não transmissíveis, e o programa de atenção ao idoso. É feita referência ao marco conceitual sobre Atenção Primária e Promoção da Saúde, dando ênfase à plataforma social que permite instrumentalizar em Cuba, as políticas universais simultaneamente com as focalizadas com base em quatro fortalezas da sociedade: a tendência a garantir a eqüidade e a justiça social, as bases criadas de acesso à educação, a interrelação do desenvolvimento do conhecimento científico e sua aplicação à saúde da população, e a promoção permanente de valores éticos solidários. Finalmente, se faz referência aos modelos de formação que se implementam na atualidade.

PALAVRAS-CHAVE: Atenção Primária; Promoção de Saúde, Cuba.

\section{INTRODUCCIÓN}

Al cumplirse 30 años de la Declaración de Alma Ata, el Informe de la Organización Mundial de la Salud de 2008 enfatizó la importancia de la Atención Primaria, evaluándola como más necesaria que nunca. Se reconoció que muchas de las causas de la falta de salud debían abordarse a través de un enfoque abarcador de la sociedad, anticipando la comprensión sobre los condicionantes que establecen la pobreza, la desigualdad y la mala alimentación. En ese contexto, se identificaba la atención primaria como política esencial, buscando adoptar una visión holística de la sociedad. El informe de la OMS de ese año reconoce que a pesar de haberse realizado importantes progresos para mejorar la salud, luchar contra la enfermedad y alargar la vida de las personas, existen significativos retos que obstaculizan el logro de tales metas, entre ellos la elevación del costo de la atención de la salud, que arroja a la Tempus - Actas de Saúde Coletiva, vol. 3, n². p. 99-111, Abr. / jun. 
pobreza o la recrudece, y acentúa la carencia de servicios para miles de personas en el mundo: "Una gran proporción de los recursos se destina a los servicios curativos, pasando por alto las actividades de prevención y promoción de la salud, que podrían reducir en un $70 \%$ la carga de morbilidad a nivel mundial. En resumen, los sistemas de salud son injustos, inconexos, ineficientes y menos eficaces de lo que podrían ser. Además, sin una reorientación sustancial es probable que los actuales sistemas de salud se vean desbordados por los crecientes desafíos del envejecimiento de la población, las pandemias de enfermedades crónicas, las nuevas enfermedades emergentes, como el SRAS, y las repercusiones del cambio climático". (OMS, 2008). Al publicar este informe la OMS pretendía iniciar un debate a nivel mundial sobre la eficacia de la atención primaria de salud, como forma de organizar el conjunto de la atención de salud, desde los hogares hasta los hospitales.

\section{ANTECEDENTES EN CUBA: FACTORES ESTRUCTURALES Y COYUNTURAS}

En los años 50 el cuadro Sanitario en Cuba se caracterizaba por el predominio de enfermedades infecciosas y parasitarias, la Mortalidad Infantil era de 60 por 1000 nacidos vivos y la expectativa de vida se estimaba en 50 años. Sólo existían en el país dos universidades, la pobreza abrumaba con su peso a la mayoría de la población, el analfabetismo era del $60 \%$. De los 6000 médicos que había en Cuba en 1959, la mayoría se encontraba en la capital, con consultas privadas, y la mitad de ellos abandonó el país al principio del período revolucionario. Este éxodo exigió un intenso proceso de formación de personal y de soluciones novedosas para enfrentar la situación. En enero de 1960 fue creado el Servicio Médico Rural, por el cual los médicos debían prestar servicios en las comunidades rurales más alejadas, sin existir todavía las carreteras ni las estructuras adecuadas para ejercer los servicios médicos. En los años siguientes se erradicaron sucesivamente diferentes enfermedades utilizando las organizaciones de masas creadas por el proceso revolucionario: ejemplo de ello fue la vacunación contra la poliomielitis, al no existir todavía el sistema de salud, se extendió el proceso de inmunización a través de los Comités de Defensa de la Revolución, así se logró erradicar la enfermedad en 1962. Progresivamente, se erradicaron el tétanos neonatal (1972), la di' eria (1979), la meningoencefalitis (1989), el sarampión (1993), la Rubéola y la parotiditis (1995), y se redujo la morbilidad de la tos ferina y el tétanos (1996), la enfermedad meningocócica, y la hepatitis B en menores de 15 años (1998)

Pero graves retos enfrenta la sociedad cubana, en primer lugar, Cuba es un país pobre, subdesarrollado, que en los años 50 se caracterizaba por el monocultivo, la comercialización del azúcar como recurso principal, y fuertes carencias económicas, asociadas a la neocolonización que se extendió en el área después de la guerra de independencia contra España. Cuando la Revolución cubana triunfó, en 1959, fue Tempus - Actas de Saúde Coletiva, vol. 3, no2. p. 99-111, Abr. / jun. 
necesario un fuerte proceso de reinserción internacional, por el inicio del bloqueo y la agresión del gobierno de los Estados Unidos, con medidas de tanto impacto económico desfavorable como el retiro de la cuota azucarera, o las presiones diplomáticas y comerciales para tratar de aislar a Cuba, lo que determinó la ruptura con el país de todos los países latinoamericanos con excepción de México. Esta situación se revirtió con los nexos que se establecieron en los años 70 con los países socialistas del este europeo.

Importantes fueron las políticas sociales instrumentadas: en los 30 años entre 1959 y 1989, la mortalidad infantil se redujo más de cuatro veces, y la escolaridad promedio alcanzó el noveno grado, creándose las bases de una sociedad equitativa, y favoreciendo la asimilación de un profundo sentimiento de igualdad y auto percepción de dignidad en la población. Entre las principales metas trazadas, se destacaba la promoción de la salud.

Pero en un lapso menor a treinta años, el país tuvo que enfrentarse por segunda vez a un nuevo reto de inserción internacional al fracasar el modelo soviético y desmembrarse el campo socialista. Este escenario se agrava al mantenerse el bloqueo por cerca de cincuenta años, con el establecimiento en los años 90 de dos leyes, la Torricelli, y la Helms Burton, que endurecen la agresión y lo extiende a terceros países, en franca violación de los principios internacionales.

Los impactos del bloqueo en la esfera de la salud se reflejan en la carencia de medicamentos., el encarecimiento del acceso a medicinas o la imposibilidad de acceso a determinados recursos como la escasa disponibilidad de placas para mamografías, que hizo necesario reducir los exámenes sistemáticos a mujeres en grupos de riesgo, o la afectación de niños con cáncer de los ojos, que no pueden acceder a medicamentos que solo se producen en los Estados Unidos..A pesar de que el rechazo a este bloqueo es prácticamente total en el mundo (la ONU ha aprobado por abrumadora mayoría una condena durante años sucesivos, sin que pueda modificarse en la práctica tales agresiones), el bloqueo persiste, y adquiere graves connotaciones.

Es sobre este escenario que debe analizarse la situación de la salud en Cuba, al enfrentarse con políticas sociales a la adversa situación. Al inicio de la crisis económica de los años 90 el sociólogo sueco Claus Brundenius, quien había investigado en la década anterior (ZIMBALIST e BRUNDENIUS, 1985) sobre el favorable comportamiento del Índice Gini en Cuba, predijo que era imposible mantener el descenso de la mortalidad infantil en el país, debido a la coyuntura adversa que sufría. Pero este pronóstico no se cumplió.

\section{CARACTERIZACIÓN GENERAL DEL SISTEMA NACIONAL DE SALUD}

Tempus - Actas de Saúde Coletiva, vol. 3, no2. p. 99-111, Abr. / jun. 
El Ministerio de Salud Pública (MINSAP) es el Organismo rector del Sistema Nacional de Salud. Es el encargado de dirigir, ejecutar y controlar la aplicación de la política del país en cuanto a la Salud Pública, el desarrollo de las Ciencias Médicas y la Industria Médico Farmacéutica. Los principios que guían el sistema de salud en Cuba son: el carácter estatal de la salud pública, (establece el deber del estado para preservar la salud de la población), la accesibilidad y gratuidad de la salud, (preserva la equidad y evita la comercialización de los servicios médicos), la orientación profiláctica, (garantiza la promoción y la prevención de la salud a escala social) ,la unidad de la ciencia, la docencia y la práctica médica, (cohesiona la unidad del sistema y garantiza su efectividad), la integralidad y el desarrollo planificado, (favorece la relación intersectorial y la equidad), la participación activa de la población, (desarrolla el protagonismo social de la población en su ejecución y proyecciones), y el principio de internacionalismo y colaboración, (expresión de los valores éticos básicos de la sociedad socialista cubana).

La estructura de la salud abarca un primer nivel de atención primaria, donde médicos y enfermeras de familia atienden alrededor de 120 familias, (500 ó 600 personas, en dependencia de la densidad de la población), con énfasis preventivo, y atención personalizada. En segundo nivel, los Policlínicos Integrales a nivel comunitario, brindan servicios de laboratorios y consultas especializadas, constituyen la plataforma especializada que se articula a los servicios del médico de familia. El tercer nivel, los hospitales, poseen mayor especialización: infantiles, ortopédicos, oncológicos, psiquiátricos, etc.. Los tres niveles se encuentran fuertemente articulados.

El médico de familia conoce los casos de pacientes de su comunidad aquejados de enfermedades crónicas, visita regularmente aquellos que requieren atención domiciliaria, y controla el estado de salud de los pacientes con hipertensión arterial, los casos de obesidad, y los discapacitados. La remisión a los policlínicos es la vía para dar continuidad a los diagnósticos iniciales, y su tratamiento especializado, incuso al nivel hospitalario cuando así se requiere.

En los últimos años, el segundo nivel, los policlínicos, ha adquirido mayor relevancia en el sistema por dos razones básicas, la necesidad de suplir cierta relativa disminución del número de los médicos de familia, muchos de los cuales prestan servicios en otros países, y la modernización, con nuevos equipamientos y ampliación de servicios que se diseñaron para la atención de la promoción de salud. Recientemente se remodelaron y ampliaron los policlínicos con equipos de alta tecnología, brindando 32 servicios a la población: ultrasonido, laboratorios integrales, salas de fisioterapia, salas de hemodiálisis, centros de terapia intensiva en las municipalidades, y se asocian 
no solo a los consultorios del médico de familia, también a las casas de abuelos, los hogares maternos y otros centros.

Sin embargo, se produce en el sistema un proceso de fortalecimiento progresivo del con-sultorio del médico de familia, clasificándolos en tres tipos, de acuerdo a su ubicación territorial, para el desarrollo de la asistencia, la docencia y la investigación. En dos de sus tres modalidades, que abarcan actualmente 10596 consultorios, se mantienen brindando servicios, diariamente en horario laboral. Los de la tercera modalidad (177 en total), clasificados como reforzados, se instalan en lugares de difícil acceso geográfico funcionando las 24 horas del día, contando con laboratorios, y servicios especializados como electrocardiograma, ultrasonidos, etc. (DE LA OSA, 2008b).

También constituyen parte de la estructura del sistema cuatro programas priorizados, de los cuales se presentan algunos de sus rasgos.

Atención Materno Infantil. Las prácticas efectivas expresan el éxito de la prevención de este programa: un ejemplo es el control sobre las pruebas citológicas del cuello del útero, que son programadas para las mujeres en edad reproductiva por los consultorios mediante sus expedientes clínicos, convocándolas por las enfermeras en los plazos correspondientes. De igual modo sucede con las consultas de las embarazadas, 95\% de ellas son captadas en el primer trimestre de gestación, y reciben 17 consultas especializadas y 30 exámenes de diagnóstico por cada embarazo. Son controladas estrechamente las consultas de puericultura en el primer año de vida del niño, que garantiza la vigilancia sobre la evolución del bebé, en peso, talla, y comportamiento, si una mamá olvida la consulta, es recordada por la enfermera, por teléfono ó visitando su casa.

Las campañas de inmunización para los niños se convocan por la prensa nacional en los períodos correspondientes a las edades de crecimiento, y reciben control sistemático desde los policlínicos, con un $99,9 \%$ de los niños protegidos a los dos años de edad contra 13 enfermedades inmunoprevenibles. Las mamografías se realizan colectivamente para mujeres en grupos de riesgo, incluyendo el arribo a la tercera edad, también convocadas por la enfermera en la comunidad.

Los indicadores de salud muestran el éxito del sistema: la mortalidad infantil en el 2008 fue de 4, 7 por mil nacidos vivos, (la más baja de la región, incluyendo a Canadá y los Estados Unidos, según UNICEF), la mortalidad de menores de cinco años es menor de 7, la expectativa de vida es de 77 años, la mortalidad materna es de 21, 3 por cien mil nacidos vivos, el $99,9 \%$ de los partos son institucionales atendidos por personal especializado.

Tempus - Actas de Saúde Coletiva, vol. 3, no2. p. 99-111, Abr. / jun. 
Control de enfermedades no trasmisibles. El perfil epidemiológico nacional se caracteriza por el predominio de la mortalidad por enfermedades crónicas no transmisibles. Las enfermedades del corazón, los tumores malignos, las enfermedades cerebro vasculares y los accidentes, representan casi las dos terceras partes de todas las defunciones que se producen en el país, siguiendo el patrón de causa de mortalidad correspondiente a países desarrollados. Además merecen mencionarse por su morbilidad la diabetes mellitus, la cirrosis hepática, el asma y la hipertensión arterial. Estas enfermedades se encuentran relacionadas con los hábitos y estilos de vida, y reciben atención preventiva y sistemática del médico de familia.

\section{CONTROL DE ENFERMEDADES TRASMISIBLES: EL SIDA.}

Un reto al que se enfrenta el país es la progresiva morbilidad de enfermedades infecciosas, vinculadas a las condiciones higiénicas del país, que aun requieren de de mayores controles, la afectación de las instalaciones hospitalarias, que a pesar de su progresiva recuperación todavía no han logrado revertir los efectos de la crisis, la presencia significativa del alcoholismo, y del habito de fumar, el sedentarismo, y la maternidad precoz. En su progresiva disminución desempeña un importante rol el medico de familia, con su labor profiláctica, así como la información a través de los medios masivos de comunicación.

En el caso del VIH, desde 1986 y hasta noviembre del 2008, fueron diagnosticadas como portadoras del virus del VIH/sida diez mil 454 personas, de las cuales 3910 han enfermado. Actualmente 8.607 personas viven con el VIH SIDA, (DE LA OSA, 2008a). El Estado cubano produce seis importantes medicamentos antirretrovirales y todos los enfermos reciben el tratamiento gratuito. El programa de lucha contra el SIDA limita las vías de contagio de la transmisión por vía sanguínea, con control y evaluaciones de la sangre, (este año se han realizado más de 1.700 mil pruebas de diagnóstico) o vertical, (solo el $3 \%$ de los bebés de madres seropositivas tiene la probabilidad de recibir la enfermedad, mediante la atención en el parto y un proceso muy fuerte de cuidado hasta que el recién nacido tiene 18 meses).

Aunque el tratamiento al SIDA se inició en los años ochenta con un obligatorio internamiento, desde hace años los enfermos pueden seleccionar si permanecen en un sanatorio con toda la atención gratuita garantizada, incluyendo alimentación, medicamentos, y hospedaje, o convivir con su familia en la comunidad. La tasa de prevalencia del VIH-Sida en Cuba es la más baja del Caribe, con un 0,1 por ciento, y figura entre los 22 países del mundo menos afectados. Sin embargo, la prevención por la via sexual no ha logrado incrementar suficientemente el uso de los condones, debido al rechazo masculino por tradiciones culturales e ideología machista predominante.

Tempus - Actas de Saúde Coletiva, vol. 3, no2. p. 99-111, Abr. / jun. 
Durante el presente año se han detectado mil 150 nuevos casos, siendo la capital del país la más afectada, el $80 \%$ de los seropositivos cubanos son hombres y de ellos, el 85 $\%$ han tenido sexo con otros hombres.

Nuevos programas de información y orientación contribuyen a la meta de prevenir la epidemia. En la actualidad la isla cuenta con unos 21.000 promotores voluntarios para informar y aconsejar sobre la epidemia, surgidos de las comunidades. Un papel relevante en la prevención lo desempeña el Centro Nacional de Prevención de las ITS/VIH/SIDA, creado hace 10 años, con una intensa labor educativa en todo el país. Entre las acciones desarrolladas está la creación de las cátedras Escuela, Salud y Sexualidad en todas las universidades pedagógicas de la isla, así como la realización de talleres de capacitación a educadores y la formación de jóvenes promotores, que ha permitido preparar para ello a miles de alumnos y profesores. De igual modo, se han distribuido en los centros educacionales de todo el país materiales sobre educación sexual y prevención del VIH y otras infecciones de transmisión sexual, paralelamente a la vigilancia epidemiológica y otras tareas de salud. Se ratifica así la concepción de que la educación es el fundamento para lograr un cambio de conducta que permita reducir el riesgo y la vulnerabilidad, las dos características que contribuyen a propagar el VIH. A nivel de propaganda nacional se destacan una telenovela de gran aceptación popular dedicada al tema, con ejemplos de casos de contagio, (La cara oculta de la luna), y un documental reciente que muestra la realidad de personas viviendo con el virus (Viviendo al Límite). El proceso de comunicación y de información se ha fortalecido progresivamente, contribuyendo a vencer los temores y estereotipos vigentes aún en la sociedad.

Atención al Adulto Mayor. Uno de los problemas más acuciantes de la sociedad cubana actual es el envejecimiento de la población, debido a la prolongación de la vida y la baja tasa de fertilidad. El 16,2 por ciento de los 11,2 millones de habitantes ya superan los 60 años, una cifra que en dos decenios debe incrementarse hasta alcanzar el 25 por ciento. La tasa de reemplazo poblacional, al menos una hija por cada madre, no se cubre desde 1978, a causa de la elevada instrucción de la mujer, sus crecientes responsabilidades profesionales. el acceso a un programa integrado de planificación familiar y la generalización del acceso y el uso gratuito y seguro de la interrupción del embarazo, lo que favoreció el importante proceso de participación social femenina. Las mujeres cubanas son el $66 \%$ de la fuerza técnica laboral, el $60 \%$ de la matrícula universitaria, y el 43, 3\% de los miembros del Parlamento. Incide además en la situación demográfica, la crisis económica iniciada en los años 90, que agudizó las condiciones de la calidad de vida, y acentuó el balance negativo de la emigración.

Ante este reto, se han diseñado programas para atender a las personas de la tercera edad, con la creación del Programa Integral del Adulto Mayor, que posee Tempus - Actas de Saúde Coletiva, vol. 3, no2. p. 99-111, Abr. / jun. 
enfoque comunitario e institucional, y se acompaña del desarrollo de la Geriatría y la Gerontología. En la actualidad existen en Cuba más de 14 mil círculos de abue-los, o grupos que se forman generalmente a nivel de barrio, ya sea para la recreación o para el cuidado durante el día. Además, se cuenta con unos 170 hogares de ancianos y con un plan de atención domiciliaria, que sirve a unas cien mil personas que viven solas. La Cátedra del Adulto Mayor, programa educativo dedicado a la vejez, e iniciado en la Universidad de La Habana, se ha extendido a otras provincias, con gran aceptación. También son incrementadas las proyecciones vitales (como la formación del club de los 120 años, que promueve parámetros de vida saludable para prolongar la existencia). Otras medidas de carácter económico social se implementan, entre ellas, el debate masivo y aprobación en el Parlamento de una nueva Ley de Seguridad Social, que modifica la edad de la jubilación, y estimula la permanencia laboral, compensando la prórroga de la edad del retiro con determinadas medidas favorecedoras y el establecimiento de un período de transición en su aplicación.

\section{MARCO CONCEPTUAL: ATENCIÓN PRIMARIA Y PROMOCIÓN DE SALUD}

La Promoción de Salud se ha definido como una estrategia, una filosofía o una forma diferente de pensar y actuar para alcanzar la salud de los pueblos. Se comprende como un enfoque cualitativamente superior al tradicional de la salud pública, un paradigma de la salud. Para OMS/OPS es indistintamente "una estrategia" o una "función esencial" de la salud pública. No obstante, a pesar de las diferentes acepciones asumidas o en debate, hay consenso en que involucra a gobiernos, organizaciones sociales y las personas, en la construcción social de la salud. (SANABRIA, 2007). Aunque el uso generalizado del concepto Promoción de salud se extendió con fuerza desde mediados del pasado siglo, todavía existe desconocimiento y limitaciones en la asimilación de su alcance y principios, así como el manejo de las herramientas que implica. Entre las limitaciones se destaca el de la comunicación, instrumento importante para influir en los cambios de la conducta, utilizado frecuentemente con enfoque reduccionista, sin su utilización coherente como parte de una estrategia de acción integral.

Para nuestro país, la salud es un derecho que tiene que ser garantizado por la sociedad y para ello se ha desarrollado una concepción integral sobre su atención, que combina las políticas de atención universal y gratuita de la salud, simultáneamente a su descentralización y atención preventiva comunitaria. Esta concepción esta acorde con la asumida por muchos especialistas:

"Las políticas públicas no son neutras, cuando se deja de invertir en un área es porque se está priorizando otra, si las políticas no explicitan como beneficiarios a los Tempus - Actas de Saúde Coletiva, vol. 3, no2. p. 99-111, Abr. / jun. 
grupos poblacionales más excluidos, se beneficiaran los grupos poblacionales más poderosos, mejor informados, más capacitados para usar la oferta pública y con mayor habilidad y capacidad organizativa para hacer valer sus derechos. Las políticas universales han mostrado avances sustantivos en mejorar coberturas, pero al mismo tiempo, han mostrado su total fracaso para romper la exclusión social, la de género, la étnica cultural. Por ello, mientras esta forma de hacer política se mantenga, las mujeres, los indígenas, los excluidos, seguirán siendo los mismos. Es necesario hacer visibles, darles rostro, edad, sexo, etnia, condición económica a los destinatarios de las políticas públicas. Esta afirmación no es equivalente a señalar que la única opción es la focalización, son necesarias políticas para la integración y movilidad social del conjunto de la población”. (DE LA ROSA, 2004, p.20-21).

La Promoción de la salud implica una concepción más amplia que la prevención, no se trata tan solo de prevenir enfermedades específicas, sino de promover en las estructuras sociales y en la ideología, las bases para garantizar la lucha contra las enfermedades, para uma vida más sana: abarca la educación como vía fundamental, la mejor nutrición, la estabilidad psíquica, la satisfacción progresiva posible en todas las esferas de la vida.

Según un especialista de salud "la crisis del Período especial provocó escasez de recursos, detonante de algunas epidemias. Los casos más conocidos fueron la de neuropatía, muy ligada a la carencia de alimentos, y las de dengue. Hubo una crisis integral del sistema, que estaba a punto de colapsar y que se reflejó en muchas cosas: en la alimentación, y por tanto en la salud; en falta de vigilancia de las condiciones de la reproducción del mosquito aedes aegypti y de la entrada del virus del dengue". (ROJAS, 2006).

Las respuestas a estos retos han logrado importantes éxitos, como la producción masiva de multivitaminas para combatir la neuropatía, que afectó a 50 mil personas en el año 2003, por el brusco descenso de la calidad de vida, o la campaña contra la propagación del dengue, que involucró a toda la sociedad y logró éxitos basándose en la participación popular, particularmente de los jóvenes.

- La plataforma social que permite instrumentar en Cuba las políticas universales simultáneamente a las focalizadas se basa en cuatro fortalezas de la sociedad:

- La tendencia a garantizar la equidad y la justicia social,

- Las bases creadas del acceso a la educación,

Tempus - Actas de Saúde Coletiva, vol. 3, n². p. 99-111, Abr. / jun. 
- La interrelación del desarrollo del conocimiento científico y su aplicación a la salud de la población,

- La promoción permanente de valores éticos solidarios.

Tendencia a la equidad. Algunos rasgos negativos se incrementaron durante la crisis económica iniciada en los años 90, como el deterioro de la calidad de vida de la población, la tendencia a la diferenciación social, cierto debilitamiento de la participación social, especialmente con los jóvenes, y la confrontación entre dos tipos de valores éticos. Pero, a pesar del adverso contexto internacional que enfrentó, mediante su inserción progresiva y sus políticas sociales, Cuba ha mantenido la tendencia hacia la equidad social.

Nuevas políticas diseñadas se contraponen a los rasgos desfavorables y permiten dar continuidad a los beneficios sociales que recibe la población, como programas sociales para impedir la exclusión y la polarización, (elevación de salarios, atención priorizada a población en desventaja social), políticas dirigidas a la participación de los jóvenes, (como la creación de la escuela de trabajadores sociales y su actuación comunitaria, programas de superación para alcanzar el grado 12, con otorgamiento de ingresos), y fortalecimiento de las bases para la continuidad en el ejercicio de los valores éticos, expresado en las campañas de salud y educación principalmente.

Expresión del carácter humanista de la salud en Cuba: en el año 2005 se realizó una encuesta nacional para el diagnóstico y atención de los discapacitados, realizándose más de 4000 estudios citogenéticas en todo el país. Más de seis mil madres han recibido su salario íntegro por cuidar a sus hijos, mientras más de 40 mil discapacitados se incorporaron al trabajo.

Se destaca el programa para niños sordos en Cuba, que han mejorado su calidad de vida con el implante coclear, operación que el Estado Cubano asume sin costo para el paciente y para lo cual invierte unos 60 mil dólares. Privativo de países de alto desarrollo, este tipo de implante comprende la inserción quirúrgica de electrodos en el oído interno, su estimulación por medios computarizados y un lento proceso de recuperación. Un total de 113 niños sordos o hipoacúsicos han recibido este implante coclear, de ellos 101 escuchan y hablan.

Acceso a la Educación. Es totalmente reconocida la importancia del acceso al conocimiento en la prevención de la salud, como es conocido, en los países más pobres, del grado de escolaridad de la madre, depende en alto grado la supervivencia de los hijos. La educación en Cuba es totalmente gratuita en todos los niveles, la población cubana posee un nivel promedio de 12 grado. Se cuenta el $100 \%$ de cobertura en la Tempus - Actas de Saúde Coletiva, vol. 3, no2. p. 99-111, Abr. / jun. 
enseñanza primaria, casi el $100 \%$ de los niños termina el 6to grado, con nuevos programas como la formación emergente de maestros primarios, estableciendo le relación de 1 maestro cada 20 alumnos. En 146 escuelas rurales estudia un solo alumno, se introdujo la computación desde la educación primaria y todas las aulas cuentan con medios de cómputo y videos que complementan la labor del maestro. El acceso a la educación superior se ha incrementado, con la descentralización de las universidades, y la incorporación de miles de jóvenes a programas de estudios garantizados: el curso escolar 2006-2007, contó con 388,495 maestros y profesores, cifra sin precedentes en la historia del sistema educacional cubano, que incluye a los mas de 120 mil profesionales universitarios que prestan servicios en la Educación superior.

En los últimos años iniciaron sus estudios 3 millones 14 mil cubanos en 13,487 centros docentes conjuntamente con 65 universidades y mas de 3000 sedes universitarias municipales, con una taza bruta de $58 \%$ de escolarización de adultos entre 18-25 años.

El país además extiende estos principios en su labor internacionalista, Cuba recibió el premio de Alfabetización de la UNESCO en Junio de 2006, por la Metodología "Yo si puedo", que ha sido implementada en 15 países.

La interrelación del desarrollo del conocimiento científico y su aplicación a la salud de la población, se evidencia con la integralidad que permite volcar los resultados de la investigación al servicio de la población, estableciéndose una relación entre investigación, producción, comercialización y disponibilidad de medicamentos a la población. (MAJOLI, 2000). La estructura de los polos científicos, (agrupación de las instituciones científicas) que reúne la mas alta calificación de los especialistas, el acceso a los recursos, la cooperación entre ellas para el servicio a la población, es uno de los ejemplos del sistema.

En 1987 se obtuvo la primer vacuna contra la Meningitis meningocócica B (hasta hoy a única en el mundo), en 1989 se obtuvo la vacuna contra la Hepatitis B, están en proceso de evaluación clínica 4 vacunas terapéuticas contra el cáncer, la primera vacuna terapéutica para el tratamiento del cáncer de pulmón avanzado fue registrada en Cuba en el año 2008 y es la única inscrita en el mundo para ese tipo de tumor maligno. Se investiga una vacuna contra el cólera, y se trabaja en obtener un inmunogeno terapéutico contra el SIDA, actualmente en fase de investigación con resultados alentadores.

El autotrasplante de células madres adultas obtenidas del mismo paciente para tratar miembros inferiores con isquemia crítica ha sido uno de los más importantes

Tempus - Actas de Saúde Coletiva, vol. 3, no2. p. 99-111, Abr. / jun. 
logros médicos cubanos, recientemente reconocido con el Premio Dionisio Daza y Chacón, que otorga la Revista Española de Investigaciones Quirúrgicas en 2007.

Se comercializan 38 productos en 40 países en el mundo, obteniendo ingresos desde los años 90 por unos 345 millones de dólares, se incluyen programas de diagnóstico prenatales, y disponibilidad de nuevos medicamentos, el Interferón (1981) la estreptoquinasa (sobrevivir al infarto cardíaco), el heberprop b (trata úlceras de pie diabético), y los anticuerpos monoclonales (utilizados en trasplantes de órganos).

Junto a esta excelencia en la labor científica aplicada a la salud, también se desarrolla y aplica en Cuba la medicina alternativa, que se practica y enseña en los centros del país.

Valores éticos. Una dimensión relevante del marco conceptual es la solidaridad, 38 mil trabajadores cubanos de la salud prestan servicios de cooperación en muchos países. Como importante ejemplo del ejercicio de estos valores, se creó en el año 2005 (con motivo de los afectados del huracán Katrina en Nueva Orleáns) el contingente Henry Reeves, para ofrecer servicios gratuitos a la población afectada, pero el gobierno de los Estados Unidos no la aceptó. El contingente, integrado por médicos y personal de salud, funciona para auxiliar a las poblaciones del mundo afectadas por desastres naturales, ha participado en varios países como Perú, México, China, Paquistán e Indonesia.

Un programa, la Operación Milagro, que impulsan Venezuela y Cuba, es hoy un ejemplo impactante en la colaboración en la salud, entre el 2004 y octubre de 2008 han sido operadas 1.314.000 personas de 33 países de América Latina, África y Asia, y para el año 2014 el objetivo del programa es llegar a la cifra de 6 millones de personas operadas. A través de este programa, personas de bajos recursos de países del Tercer Mundo son operadas de manera gratuita de enfermedades de la visión, como cataratas, glaucoma, estrabismo y otras.

La formación de valores éticos es una vía crucial para desarrollar la promoción de la salud, en una concepción que destina los servicios de salud a los seres humanos, como un derecho para preservar y potenciar la existencia.

\section{MODELOS DE FORMACIÓN}

Las Ciencias Médicas abarcan las carreras de Medicina, Estomatología y las licenciaturas en Enfermería, Tecnología y Psicología de la Salud, funcionan cuatro Institutos Superiores de Ciencias Médicas (en Ciudad de La Habana, Santiago de Cuba, Camagüey y Villa Clara) y 24 facultades de Medicina, cuatro de Estomatología, e igual Tempus - Actas de Saúde Coletiva, vol. 3, no2. p. 99-111, Abr. / jun. 
número de Enfermería y Tecnología, además de 30 filiales de Ciencias Médicas en todas las provincias del país. También 33 facultades para la formación de estudiantes del Nuevo Programa de Formación de Médicos Latinoamericanos y facultad de Enfermería, que se suman al programa de la Escuela Latinoamericana de Medicina (ELAM). La matrícula del curso académico 2008-2009 asciende a 200000 estudiantes de Ciencias Médicas, en todos los años de las carreras. De ese total, 173903 son alumnos cubanos, y el resto proceden de otros países.

Se ha realizado una importante transformación en el modelo de formación médica mediante el proceso de conversión en docentes de los hospitales nacionales, provinciales y municipales, programa que se extendió a los policlínicos. De este modo, la política de formación se dirige a la descentralización, con un saldo altamente favorable, vinculando la asistencia a la labor docente: la formación transcurre dentro del sistema de salud, con la presencia masiva de docentes en todo el territorio nacional, transformando el policlínico en una sede universitaria, existen en el país 200 sedes universitarias a este nivel.

Los estudiantes tienen diferentes vías para ingresar, incluso las matrículas se encuentran también municipalizadas, de acuerdo a las necesidades de los territorios, y buscando la identificación de los estudiantes con sus comunidades, incrementando el sentido de pertenencia, y vinculando su práctica y su estudio al escenario de la atención primaria.. Este modelo de formación es mucho más flexible, y se caracteriza por la utilización de nuevas tecnologías, como la integración de asignaturas, y la utilización de clases magistrales grabadas.

Estos programas se relacionan con el programa de colaboración internacional, donde los colaboradores en otros países son también profesores y ejercen su docencia en 71 países. La universalización de la enseñanza médica ha ido acompañada de un proceso de categorización docente, ello permite que se cuente con más de 26000 profesores en Ciencias Médicas que alcanzan un alto nivel académico y profesional, lo que posibilita también una mejor asistencia médica a la población.

Uno de los programas de mayor relevancia llevado a cabo en el terreno de la salud integrada a la concepción internacionalista es la Escuela Latinoamericana de Ciencias Médicas (ELAM), creada en 1988 por iniciativa del líder Fidel Castro, para formar de modo gratuito a médicos de otros países, con el compromiso de regresar a sus países de origen y ejercer la medicina solidaria en los lugares más recónditos y principalmente para los pobres. Cada año esta escuela ofrece mil 500 becas para formar gratuitamente médicos, en el año 2008 posee una matrícula de aproximadamente 10 mil estudiantes de casi 30 países, procedentes de países latinoamericanos, africanos y un grupo de estudiantes de Estados Unidos. Actualmente se desarrolla también esta Tempus - Actas de Saúde Coletiva, vol. 3, no2. p. 99-111, Abr. / jun. 
formación en sus respectivos países, al formarse los especialistas de Medicina General Integral en el último año en su territorio de origen, bajo la asesoría de los especialistas cubanos. Desde el periodo 2005-2008 Cuba ha formado seis mil 757 profesionales de la salud de 56 naciones, mediante el proyecto de la ELAM y el Convenio del Ministerio de Relaciones Exteriores de Cuba. En el curso escolar 2007-2008 egresaron mil 824 jóvenes de otros países, de ellos mil 500 de la ELAM y el resto por los convenios del MIN-REX.

La formación postgraduada se desarrolla también en fusión académico formativo y asistencial, con el desarrollo de programas de especialidades, maestrías, y doctorados.

El médico es el eslabón que trasciende la formación científica hacia la integralidad de un ser humano mejor: al prestar sus servicios en otros escenarios, realizan importantes practicas y aprendizajes sobre enfermedades que no existen en Cuba, pero simultáneamente, al enfrentar situaciones sociales mas adversas, y no disponer de un sistema de apoyo, desarrollan sus capacidades profesionales y potencian sus valores .personales.

\section{BIBLIOGRAFÍA}

ALBURQUERQUE, F. Desarrollo económico local y descentralización en América Latina. En: Revista de la CEPAL 82 Abril. 2004.

CIEM. Investigación sobre desarrollo humano y equidad en Cuba 1999. La Habana: Editorial Caguayo; 2000. Cap. 9.

DE LA OSA, J. Fructífero intercambio en temas de salud. Periódico Granma, 25 diciembre 2008a, p. 5.

La prevención en nuestras manos, Periódico Granma, La Habana. 1 diciembre de 2008b, p. 3.

DE LA ROSA, L. Descentralización de la provisión de servicios de salud: una oportunidad para el empoderamiento de las mujeres y la transversalización de la perspectiva de equidad de género. Organización Panamericana de la Salud (OPS), 2004.

DÍAZ, E. Is the Socialist Model Still a Viable Alternative in Cuba? En: BELL LARA, J. y DELLO BUONO, R. Cuba in the 21 century. Realities and Perspectives. La Habana: Edito-rial José Martí. 2005.

Tempus - Actas de Saúde Coletiva, vol. 3, no2. p. 99-111, Abr. / jun. 
Marxismo y salud. FLORENTINO, M. e SILVA, M.D.F. (Org.). Recortes do Pensamento Marxista em Saúde Seminario. Edição foi independente. Brasília: Dreams grafica, NESP/CEAM/UnB. 2006.

Impactos del desarrollo científico en la salud y la calidad de vida del género femenino en Cuba. Logros y desafíos. En VI Congreso de Ciencia, Tecnología y Género. Prensa Universitaria de Zaragoza. 2006.

Políticas Públicas en América Latina e igualdad de oportunidades. En: Diálogos sobre la Patria Grande, contribuciones de los ocupantes (2004/2005) a la Cátedra de Estudios Vilmar Faría de Estudios Latinoamericanos. FLACSO Brasil, Brasilia. 2006.

DÍAZ, E.; ESPINOSA, E. Cuba: Experiencia de Desarrollo social Ético y Democracia Participativa. En La Integración Latinoamericana. Versiones Regionales y Subregionales. FLACSO Secretaría General, San José, OMS (2008) Informe de salud. 2006.

El desarrollo del sistema nacional de salud en Cuba. En: Salud para todos si es posible. Sociedad cubana de salud pública. La Habana, Sección de Medicina Social. 2005. p. 39-70.

MAJOLI, M. Ciencia, tecnología y desarrollo. La industria bio tecnológica cubana: una aproximación. Tesis doctoral. Programa FLACSO Cuba MINSAP (2007) Anuario Estadístico de Salud. 2000.

PÉREZ, C. Caring for them from birth to death: the Ethics, Ideologies, values and practices of cuban medicine. Tesis de Doctorado, Universidad de Chicago. 2004.

ROJAS, F. Modelos de salud en Cuba. Habla un protagonista. (entrevista al Doctor Francisco Rojas Ochoa por Rodríguez Lázaro). En: Revista Temas No. 47 Julio septiembre, La Habana. 2006.

SANABRIA, G. El debate en torno a la promoción de la salud y la educación para la salud. Revista Cubana Salud Pública, 33, La Habana. 2007.

ZIMBALIST, A.; BRUNDENIUS, C. Crecimiento con equidad: el desarrollo cubano en una perspectiva comparada. Cuadernos de Nuestra América. La Habana, vol VI, No. 13 (julio, diciembre). 1985.

Tempus - Actas de Saúde Coletiva, vol. 3, no2. p. 99-111, Abr. / jun. 